\title{
GESTIÓN DE RESIDUOS SÓLIDOS EN CADENAS DE SUMINISTRO DE CICLO CERRADO DESDE LA PERSPECTIVA DE LA INVESTIGACIÓN DE OPERACIONES
}

\author{
Claudia Cecilia Peña-Montoya ${ }^{1}$ \\ Juan Carlos Osorio-Gómez ${ }^{2}$ \\ Carlos Julio Vidal-Holguín ${ }^{3}$ \\ Patricia Torres-Lozada ${ }^{4}$ \\ Luis Fernando Marmolejo-Rebellón ${ }^{5}$
}

Recibido el 20 de marzo de 2014, aprobado el 29 de septiembre de 2014 y actualizado el 27 de abril de 2015

DOI: 10.17151/luaz.2015.41.2

\section{RESUMEN}

La integración de los flujos reversos y directos en la cadena de suministro, trae beneficios al reducir costos y evitar el daño ambiental porque se recupera valor de los residuos sólidos; esta integración se conoce como la cadena de suministro de ciclo cerrado. La mayoría de experiencias que relacionan cadena de suministro de ciclo cerrado y decisiones de gestión de residuos sólidos, provienen de países desarrollados con estrictas regulaciones ambientales y usan técnicas de invetigación de operaciones. El objetivo se este artículo es identificar la factibilidad de aplicar técnicas de investigación de operaciones a las decisiones de gestión de residuos sólidos en las cadena de suministro de ciclo cerrado en países en desarrollo como Colombia. Se identificaron variables importantes de los articulos revisados tales como los diferentes tipos de residuos, las regiones y el uso de técnicas de optmización, simulación y multicriterio. La metodología de vigilancia tecnológica se uso para correlacionar estas variables. Los resultados mostraron que la técnica de multicriterio es usada con más frecuencia porque permite la consideración de varios aspectos en una misma decisión; por lo tanto, el uso de esta técnica sería conveniente para enfrentar efectivamente las principales decisiones en las incipientes cadenas de suministro de ciclo cerrado en países en desarrollo.

\section{PALABRAS CLAVE}

Cadena de suministro, ciclo cerrado, análisis multicriterio, optimización, residuos sólidos, simulación.

\section{SOLID WASTE MANAGEMENT IN CLOSED-LOOP SUPPLY CHAINS FROM THE PERSPECTIVE OF OPERATIONS RESEARCH}

\begin{abstract}
The integration of reverse and direct flows within the supply chain is highly benefitial to reduce costs and avoid the enviromental damage because value from the solid waste is recovered; this
\end{abstract}


integration is known as closed-loop supply chain. Most of the reported experiences regarding closed-loop supply chains and solid waste management decisions come from developed countries with strict environmental regulations and use of operations research techniques. This paper aims to identify the feasibility of applying operations research techniques to solid waste magement decisions within closed-loop supply chains in developing countries such as Colombia. Important variables such as the different kinds of solid waste, the regions and the use of optimization, simulation and multi-criteria techniques were identified by reviewing research articles. Technologic watch methodology was used to correlate these variables. The results showed that the multi-criteria technique is most frequently used because it allows including different aspects related to a single decision; therefore, the use of this technique would be appropiate to effectively approach the main decisions at the emerging closed-loop supply chains in developing countries.

\section{KEY WORDS}

Supply chain, closed loop, multicriteria analysis, optimization, solid waste, simulation.

\section{INTRODUCCIÓN}

El incremento en la generación y disposición final de residuos sólidos tiene múltiples efectos en la calidad de vida, conservación del ambiente y ganancia de las industrias. Según se define en el Decreto 2981 (Ministerio de Vivienda, Ciudad y Territorio, 2013) residuo sólido:
Es cualquier objeto, material, sustancia o elemento principalmente sólido resultante del consumo o uso de un bien en actividades domésticas, industriales, comerciales, institucionales o de servicios, que el generador presenta para su recolección por parte de la persona prestadora del servicio público de aseo.

Así mismo, este decreto define la gestión integral de residuos sólidos (GIRS):

Es el conjunto de actividades encaminadas a reducir la generación de residuos, a realizar el aprovechamiento teniendo en cuenta sus características, volumen, procedencia, costos, tratamiento con fines de valorización energética, posibilidades de aprovechamiento y comercialización. También incluye el tratamiento y disposición final de los residuos no aprovechables. 
El aumento de precio de los combustibles fósiles y materia prima, el incremento de productos manufacturados desechados que ocupan los rellenos sanitarios, la regulación y la reducción de terrenos disponibles para ser usados como rellenos sanitarios, son aspectos que motivan a recuperar valor de los productos y disponerlos adecuadamente (Ferguson y Souza, 2010). El interés en la recuperación de materiales ha hecho que el alcance de la cadena de suministro directa, involucre también el canal de reversa para integrar la cadena de suministro de ciclo cerrado (CSCC), las cuales son cadenas de suministro (CS) donde además del típico flujo hacia adelante de materiales e información desde los proveedores hasta los consumidores, también hay flujos de productos hacia atrás desde los consumidores hasta los fabricantes (Ferguson y Souza, 2010).

De acuerdo con Talbot et al. (2007), la importancia de integrar las cadenas radica en que las iniciativas ambientales se maximizan si se integran en una configuración estratégica y por el contrario, se minimizan si se toman por separado en un nivel táctico. La gestión de la CSCC es compleja porque hay mayor número de actores y de actividades que en la CS tradicional (Thiell et al., 2011).

Como lo muestra la Figura 1, la gestión de la CSCC comprende dos dimensiones que involucran la gestión de flujo de materiales e información al interior de las organizaciones: Logística Verde (LV) y Logística de Reversa (LR); la LV es una estrategia para optimizar los consumos de materiales, reducir los desperdicios y adoptar prácticas de logística amigables con el ambiente y además favorece el desempeño económico de la organización, mejora el de la cadena de valor y aporta elementos para el bienestar social (Thiell et al., 2011). La LR es el proceso de trasladar bienes desde su típico destino final hacia opciones que permitan capturar valor o garantizar la disposición final adecuada (Rogers y Tibben-Lembke, 1998).

Según Ferguson y Souza (2010), las decisiones que enfrentan las CSCC son de diversa índole, dependen de los objetivos de la organización y del tipo de retornos que manejen; por ejemplo retornos de los consumidores, retornos de fin de uso o retornos de fin de ciclo de vida. Así mismo, las decisiones mencionadas dependen de las diferentes formas de gestión de los materiales retornardos. En la Tabla 1 se presentan algunas decisiones comunes en las CSCC. Las decisiones estratégicas en las CSCC dan lugar a la mayoría de impactos financieros y ambientales (Souza, 2013). En el aspecto financiero es necesario realizar inversiones en infraestructura, en capacitación del personal y esfuerzos de mercadeo adicionales para reintregar los productos recuperados al mercado. En el aspecto ambiental, se busca el cumplimiento de la legislación, el rediseño de productos o procesos con nuevas características, la disminución de materiales aprovechables que se conducen a los rellenos sanitarios y la reducción de emisiones y lixiviados (GranadaAguirre, 2009). 


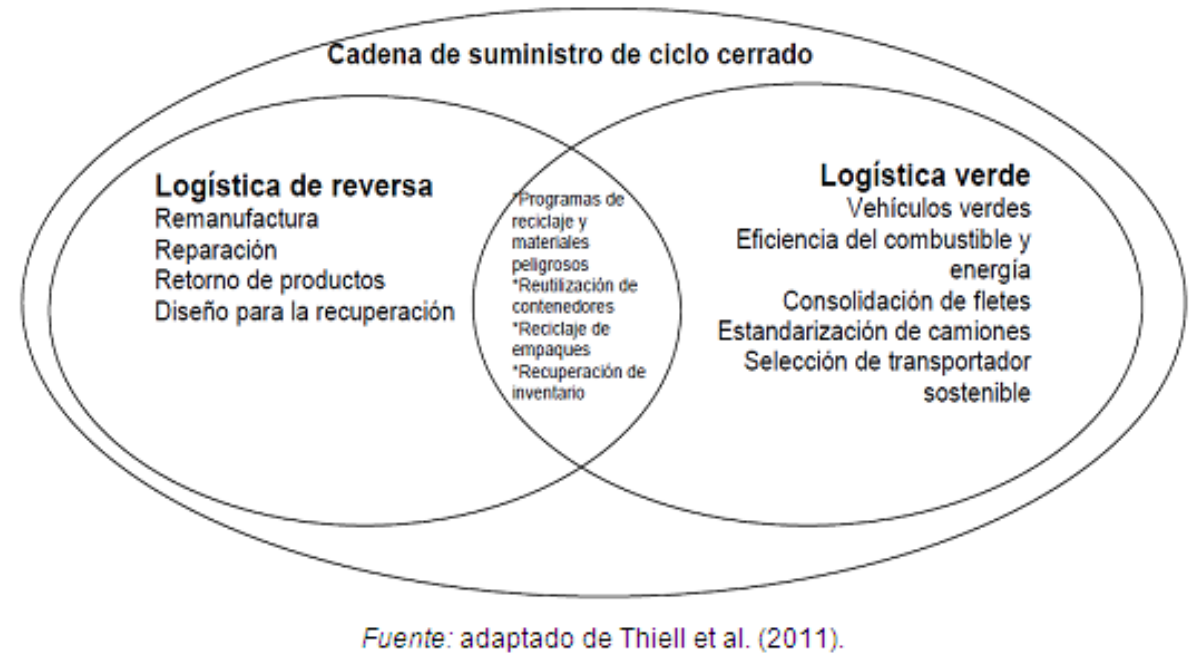

Figura 1. Relaciones entre CSCC, LV y LR.

Tabla 1. Niveles y decisiones en las cadenas de suministro de ciclo cerrado

\begin{tabular}{ll}
\hline Nivel & \multicolumn{1}{c}{ Decisiones } \\
\hline Diseño de la red: localización y tamaño de los \\
centros de recolección, instalaciones de \\
remanufactura. \\
Estrategia de recolección: ¿los consumidores \\
deben devolver los productos a los minoristas o \\
directamente a los fabricantes? \\
¿Los fabricantes deben remanufacturar? \\
Coordinación de la cadena de suministro: \\
contratos e incentivos. \\
Responder a la legislación de recuperación. \\
Impacto de las actividades de recuperación en el \\
diseño de nuevos productos. \\
Adquisición de retornos de productos: ¿cuántos, \\
cuándo y de qué calidad? \\
Disposición de devoluciones: remanufactura, \\
desmantelamiento de partes sobrantes o \\
reciclaje. \\
Planeación del desensamble, secuencia y \\
profundidad del desensamble. \\
Programación, reglas de prioridad, tamaño del \\
lote y rutas en el almacén de remanufactura. \\
\hline
\end{tabular}

Fuente: adaptado de Souza (2013).

Para abordar aspectos complejos de las decisiones en CSCC, se usan las técnicas de investigación de operaciones, las cuales, según Stindt y Sahamie (2014), deben utilizar modelos a ser desarrollados considerando las circunstancias de cada sector en 
la industria y el nivel de la decisión a tomar. Entre las técnicas más conocidas para respaldar las decisiones de las CSCC, se encuentran las de optimización, simulación y análisis multicriterio.

El objetivo de este artículo es reflexionar acerca de las oportunidades de aplicación de las técnicas de investigación de operaciones GIRS en países en desarrollo como Colombia, a través de la revisión y sistematización de la literatura científica que relaciona las técnicas de investigación de operaciones con la gestión de residuos sólidos y las cadenas de suministro de ciclo cerrado. Para tal fin, se presenta la revisión de literatura en las técnicas de investigación y posteriormente la metodología usada para el cumplimiento del objetivo. En las secciones finales se encuentran los resultados y discusión y las conclusiones del estudio.

\section{TÉCNICAS DE INVESTIGACIÓN}

\section{Optimización}

La intención de la optimización es identificar el máximo o mínimo relativo de una función, lo cual se logra a través de modelos de programación matemática, que formulan varias restricciones y una función objetivo; esta información puede ser implementada para mejorar el desempeño de la compañía en la cadena de suministro (Shapiro, 2007). Los modelos de optimización son usados principalmente para respaldar decisiones estratégicas y tácticas de las cadenas de suministro (Shapiro, 2007) y en las CSCC son usados generalmente para definir la configuración de la cadena, es decir, determinar la ubicación y número de instalaciones, la capacidad y los flujos de materiales que hay entre estas (Casas y Cerón, 2013). Ejemplos típicos incluyen la programación lineal para funciones objetivo y restricciones lineales, la programación entera para soluciones enteras, así como la programación entera mixta (Dale et al., 2012).

Tradicionalmente, los modelos para el diseño de la CS se enfocan en un solo objetivo y se utiliza como medida de desempeño la minimización de costos o la maximización de utilidades; sin embargo, hay otras medidas como maximizar el nivel de servicio al cliente, minimizar los riesgos financieros y maximizar la calidad de los productos (Ramezani et al., 2013). El diseño de la CSCC se ha llevado a cabo principalmente a través de modelos determinísticos y con un único objetivo (Fleischmann et al., 2000; Demirel y Gökçen, 2008; Zhou y Wang, 2008; Gomes-Salema et al., 2010; Lu y Zhao, 2010; Paksoy y Bektas, 2011; Paksoy et al., 2011) y ha tomado importancia porque la gestión integrada de la CS directa y la inversa, resulta en mejor desempeño global (Pishvaee et al., 2011); sin embargo, para evitar la suboptimización como resultado del diseño por separado de las dos cadenas, algunos autores como Gomes-Salema et al. (2010) han formulado modelos de optimización que consideran el diseño integrado de la CSCC. 
De acuerdo con Yeomans (2007), los modelos de optimización en CSCC, aplicados a situaciones de gestión de residuos son apropiados para problemas bien estructurados; por tanto, los componentes de incertidumbre presentes en la gestión de residuos sólidos limitan su implementación práctica. La incertidumbre se aborda desde los modelos de optimización estocásticos; sin embargo, estos modelos enfrentan las limitaciones de disponibilidad de datos históricos para estimar las distribuciones de probabilidad para los parámetros no determinísticos y la gran cantidad de escenarios usados para representar la incertidumbre dan lugar a largos tiempos computacionales (Pishvaee et al., 2011). Son varios los autores que han utilizado la programación estocástica para la consideración de la incertidumbre en la configuración de CSCC (Gomes-Salema et al., 2007; Pishvaee et al., 2009; Lee et al., 2010).

Además, el diseño de las CSCC implica compensaciones entre los diferentes objetivos que son incompatibles (Wang et al., 2011). Según Ramezani et al. (2013), en el diseño de cadenas es irreal considerar un solo objetivo como minimizar los costos o maximizar la utilidad y se deben considerar objetivos asociados a impactos ambientales o incluso impactos sociales. En cuanto a modelos multiobjetivo para la CSCC, algunos autores plantean combinación de objetivos como minimizar los costos totales del sistema y maximizar el uso de materiales y tecnologías amigables con el ambiente (Amin y Zhang, 2013); satisfacer las expectativas de precios de los clientes, detallistas y distribuidores; maximizar la satisfacción de los clientes y maximizar la utilidad (Özkır y Başıgil, 2013), maximizar la utilidad de la cadena, maximizar el nivel de servicio al cliente e incrementar la calidad al minimizar el número total de defectos en las materias primas adquiridas de los proveedores (Ramezani et al., 2013).

\section{Simulación}

Comprende enfoques como la simulación de eventos discretos (SED), la dinámica de sistemas (DS), simulación Montecarlo, Redes de Petri y modelación basada en agentes; la simulación se usa para entender cómo los sistemas se comportan en el tiempo y comparar su desempeño bajo diferentes condiciones (Tako y Robinson, 2012). De acuerdo con Tako y Robinson (2012), no hay evidencia que sugiera la correlación entre el nivel de las decisiones a estudiar (estratégica, táctica y operativa) y el enfoque de simulación a usar para situaciones de las CS; sin embargo, según Besiou et al. (2012), un enfoque de uso creciente es la DS, la cual permite aproximarse al comportamiento de problemas con complejidad dinámica como es el caso de las decisiones en las CSCC.

Los estudios de diseño de la CSCC vía simulación son escasos comparados con los estudios que usan optimización; porque cuando se utiliza simulación, no se encuentra una solución 
óptima, pero a cambio de esto, se encuentran varias soluciones cercanas a las óptimas, lo que brinda mayores posibilidades para seleccionar la que se ajuste más a la realidad (Jianghong, 2010). La mayoría de estudios de diseño de la CSCC relacionados con simulación muestran que esta herramienta es muy útil cuando la CSCC está previamente diseñada y sirve como un complemento de los modelos de optimización para evaluar su desempeño, ya que permite incorporar datos estocásticos que imitan a la naturaleza probabilística del sistema en consideración (Biehl et al., 2007; Xianfeng et al., 2010). Hay otro tipo de estudios que involucran simulación en el diseño de la CSCC, los cuales son híbridos porque utilizan la simulación junto con otra herramienta, como modelos de optimización, para realizar la evaluación del diseño previo, obtenido por optimización (Shi et al., 2010; Xianfeng et al., 2010).

\section{Multicriterio}

El análisis de decisión multicriterio (ADMC) comprende una diversidad de métodos, enfoques y técnicas para ayudar a la toma de decisiones en términos de escoger, categorizar o clasificar acciones. La base de este análisis es un conjunto finito o infinito de acciones (alternativas, soluciones), al menos dos criterios y un decisor (Figueira et al., 2005).

Hay pocos estudios de técnicas multicriterio para el diseño de la CSCC; la mayoría de ellos son discretos y evalúan alternativas de decisión definidas anteriormente desde diversos criterios, como económicos, sociales y ambientales (Casas y Cerón, 2013). Se requiere un estudio previo para definir las alternativas, los criterios y los pesos o importancia dada a cada uno de los criterios para posteriormente abordar el diseño de CS. El ADMC se ha usado en el diseño de la CSCC (Barker y Zabinsky, 2011), la localización de plantas de reprocesamiento (Queiruga et al., 2008; Achillas et al., 2010; Aragonés-Beltrán et al., 2010), la selección de modo de gestión de residuos (Rousis et al., 2008; Banar et al., 2010); las decisiones de operadores de gestión de residuos (Senthil et al., 2012). Para abordar estas decisiones, se usan enfoques basados en la utilidad multiatributo como el ELECTRE, PROMETHEE, GAIA, AHP y ANP los cuales presentan ventajas y desventajas según la aplicación en la GRS (Pires et al., 2011).

\section{METODOLOGÍA}

Para el desarrollo del objetivo propuesto, se usó la metodología de vigilancia tecnológica que consiste en realizar de forma sistemática la captura, el análisis y la difusión de las informaciones técnicas útiles para el desarrollo de la investigación y comprende cuatro etapas básicas (Escorsa y Maspons, 2001). La primera etapa, de recuperación de la información y registro en una base de datos, consiste en consultar bases de datos de información técnica según el área que se está investigando; también hay motores de búsqueda 
especializados en documentos académicos. El registro de la información varía de acuerdo con las necesidades de los investigadores y puede comprender el título, las palabras clave, el país y otra información relevante para la investigación. Para este caso se efectuó la recuperación hasta diciembre de 2012.

La segunda etapa es el análisis de frecuencias que comprende el conteo de las palabras que se encuentran en diferentes campos como los títulos, resúmenes y palabras clave. Básicamente da cuenta de la frecuencia en que algunas palabras que interesan a la investigación, aparecen en la información recuperada.

La tercera etapa es la coocurrencia de palabras expresa cuando dos palabras van juntas en el título, resumen, en las palabras clave o en secciones que interesan para la investigación. Si la coocurrencia es elevada, significa que hay una proximidad, que dos palabras tienen relación. Si la coocurrencia es baja, quiere decir que no hay relación y la última etapa se realiza con base en los criterios de proximidad y distancia de los datos, a traves de lo cual es posible representar un campo determinado en forma de gráfico y esto da lugar a los mapas tecnológicos.

\section{RESULTADOS Y DISCUSIÓN}

\section{Recuperación de la información y registro en una base de datos}

Se partió de la información disponible en las bases de datos como ScienceDirect, Scopus, Ebsco, Jstor y Emerald Insight; además, se consultaron documentos disponibles en el buscador académico Google Scholar. También, se recurrió a documentos impresos como tesis y artículos disponibles en la biblioteca de la Universidad del Valle. Las palabras clave usadas para la recuperación se muestran en la Tabla 2.

Tabla 2. Palabras clave para la recuperación de artículos

\begin{tabular}{ll}
\hline Palabras clave principales & Otras palabras clave \\
\hline Closed-loop supply chain & Green supply chain \\
Solid waste management & Green logistics \\
Operations management & Reverse logistics \\
Optimization & Sustainable supply chain \\
Simulation & Sustainable solid waste management \\
Multicriteria Decision Analysis & Reverse supply chain \\
\hline
\end{tabular}

Fuente: los autores.

Se usaron combinaciones de las palabras clave para recuperar la información. Una vez recopilada, se procedió a su lectura identificando aspectos principales y registrándolos en una base de datos. Aspectos de interés fueron el título, los autores, el año, las palabras clave, el tipo de residuos, la técnica de investigación 
de operaciones y el software. Se recuperaron inicialmente 180 artículos; sin embargo, después de su lectura se definieron 47 artículos como los más relevantes para la investigación porque involucraban aspectos de la GIRS y de CSCC.

Las Tablas 3 a la 5 muestran el registro de la información según la técnica de investigación de operaciones, es decir optimización, simulación y multicriterio, siendo estos últimos los más numerosos (27), seguidos por los de optimización (13) y finalmente los de simulación (7). En esta última categoría predominan los modelos de DS. En la Tabla 6 se aprecian las convenciones usadas en la Tabla 5 correspondiente a la técnica multicriterio.

\section{Análisis de frecuencias}

En esta sección se analizaron las aspectos generales de cada una de las técnicas de investigación de operaciones. Las Tablas 7 a la 11 resumen las frecuencias de los aspectos de interés para el estudio. En la Tabla 7 se aprecia el aumento del número de artículos que involucran la GIRS y la CSCC a partir del año 2008, lo que demuestra la inclinación de los investigadores por incluir estos temas entre sus intereses. Los residuos sólidos municipales tuvieron la mayor participación al comparar las tres técnicas; sin embargo, estos correspodían a modelos multicriterio en su totalidad (Tabla 8). Seguidamente se encontraron aquellos artículos que no especificaban (NE) el tipo de residuo para el cual estaba dirigida la investigación, lo que supone que el modelo era planteado para abordar decisiones en CSCC independiente del tipo de residuo.

Las herramientas utilizadas varían según el tipo de investigación; además se identificó una tendencia a usar híbridos, es decir la combinación de dos o más técnicas para abordar aspectos como la incertidumbre y la complejidad de las CSCC (Tabla 9). Este comportamiento era más marcado en la técnica multicriterio donde por ejemplo se encontró combinación de herramientas cono AHP, TOPSIS y FUZZY.

Los software para resolver los modelos son propios de cada técnica, por ejemplo para los modelos de optimización predominó el uso de GAMS y CPLEX; para los de simulación el POWERSIM y para los multicriterios no era usual mencionar el software utilizado (Tabla 10). Turquía, Canadá y Grecia fueron los países de los que provenían la mayoría de los artículos consultados (Tabla 11). Específicamente en los temas de la investigación no se hallaron artículos que hicieran referencia a países de América Latina. 
Tabla 3. Modelos de Optimización

\begin{tabular}{|c|c|c|c|c|c|c|c|}
\hline \multirow{14}{*}{ 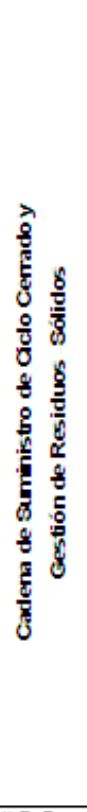 } & No. & Autores & Ano & Pals & Software & Residuos & Herramlenta \\
\hline & 1 & Pls hvaee et $3 \mathrm{l}$. & 2009 & Iran & LINGO 8.0 & NE & $\begin{array}{l}\text { ESTOC+ } \\
\text { MILP }\end{array}$ \\
\hline & 2 & Paksoy et 3 l. & 2010 & Turquía & UNDO 6.1 & NE & $\begin{array}{l}\mathrm{ILP+} \\
\mathrm{MOB}\end{array}$ \\
\hline & 3 & $\begin{array}{c}\text { Gomes-Salems } \\
\text { et al. }\end{array}$ & 2010 & Portugal & $\begin{array}{l}\text { GAMS } \\
\text { CPLEX }\end{array}$ & VIorio & MILP \\
\hline & 4 & Kannan et al. & 2010 & Indis & $\begin{array}{l}\text { Algoritmos } \\
\text { geneticos y } \\
\text { G.AMS }\end{array}$ & Baterlas & MILP \\
\hline & 5 & Paksoy et 3 l. & 2011 & Turquía & LINDO 6.1 & NE & MINLP \\
\hline & 6 & Znang \& AmIn & 2011 & Canada & CPLEX 9.1 & Fotocopladora & $\begin{array}{l}\text { MILP+ } \\
\text { FUZZYY }\end{array}$ \\
\hline & 7 & Plshvaee et al. & 2011 & Iran & $\begin{array}{c}\text { ILOG } \\
\text { CPLEXX } \\
10.1\end{array}$ & NE & $\begin{array}{l}\text { MILP+ } \\
\text { ROBUSTO }\end{array}$ \\
\hline & 8 & Amin $\&$ Znang & 2013 & Canaoa & CPLEX 9.1 & Fotocopladora & $\begin{array}{l}\text { MILP+MOB+ } \\
\text { ESTOC }\end{array}$ \\
\hline & 9 & Zeos\|cs et 3 al. & 2012 & Portugal & $\begin{array}{l}\text { GAMS } \\
\text { CPLEX }\end{array}$ & Varlos & MILP \\
\hline & 10 & AmIn \& $z$ nang & 20123 & Canaca & GAMS & computadores & $\begin{array}{l}\text { FUZZY A.HP + } \\
\text { MOB MILP }\end{array}$ \\
\hline & 11 & OzkIr \& Bas\|g\| & 2013 & Turquia & $\begin{array}{l}\text { GAMS } \\
\text { BARON }\end{array}$ & NE & $\begin{array}{l}\text { MOE+ } \\
\text { FUZZYY }\end{array}$ \\
\hline & 12 & Amin $\& Z$ nang & 20120 & Canacas & GAMS & computadores & MILP \\
\hline & 13 & Mitra 3 & 2012 & Indla & $\begin{array}{l}\text { SLAM II } \\
\text { (SImulation } \\
\text { Language } \\
\text { for } \\
\text { Alter natlve } \\
\text { Modelling) }\end{array}$ & Fotocoplador 3 & $\begin{array}{c}\text { DETERM+ESTOC } \\
\text { WARNER } \\
\text { WHITING } \\
\text { ALGORITHM }\end{array}$ \\
\hline \multicolumn{3}{|c|}{ 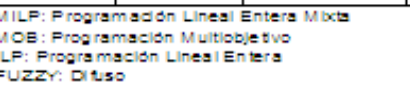 } & & \multicolumn{4}{|c|}{ 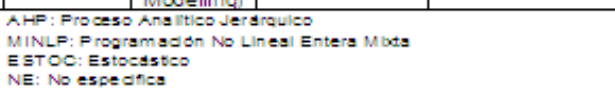 } \\
\hline
\end{tabular}

Tabla 4. Modelos de Simulación

\begin{tabular}{|c|c|c|c|c|c|c|c|c|c|c|}
\hline \multirow{9}{*}{ 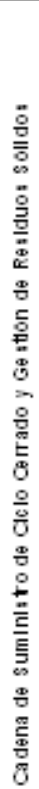 } & \multirow{2}{*}{$\mathrm{Nan}$} & \multirow[b]{2}{*}{ Anomen } & \multirow[b]{2}{*}{ na } & \multirow[b]{2}{*}{ Pais } & \multirow[b]{2}{*}{ sactwore } & \multirow[b]{2}{*}{ Aladons } & \multicolumn{3}{|c|}{ Simudadion } & \multirow[b]{2}{*}{ ora } \\
\hline & & & & & & & 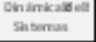 & 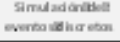 & 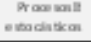 & \\
\hline & 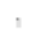 & 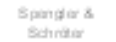 & 200 & Alamaria & VENSAM & RNEE & 1 & & & \\
\hline & 2 & Sorrabtat. & nes & saphen & ouEst & NE & & 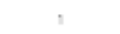 & & \\
\hline & 3 & Matios at a. & 200 & Cоmas & 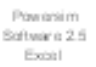 & NE & 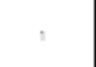 & & & \\
\hline & 4 & $\begin{array}{c}\text { Compzadsis } \\
\text { Bessiou }\end{array}$ & 2000 & Cnasa & Pon iarame & RNEE & 1 & & & \\
\hline & s & Froding at a. & $20 \div 0$ & Alamaria & М⿻pen Рus & Zne & & & & 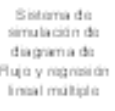 \\
\hline & a & Beam ital al & 2010 & Спзаз & NE & RNEE & $\pi$ & & & \\
\hline & 7 & Wing S Minata & 2011 & saptor & Promisaim & ve & 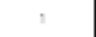 & & & \\
\hline
\end{tabular}

Fuente: los sutores.

Hacer click en la imagen para ampliarla 


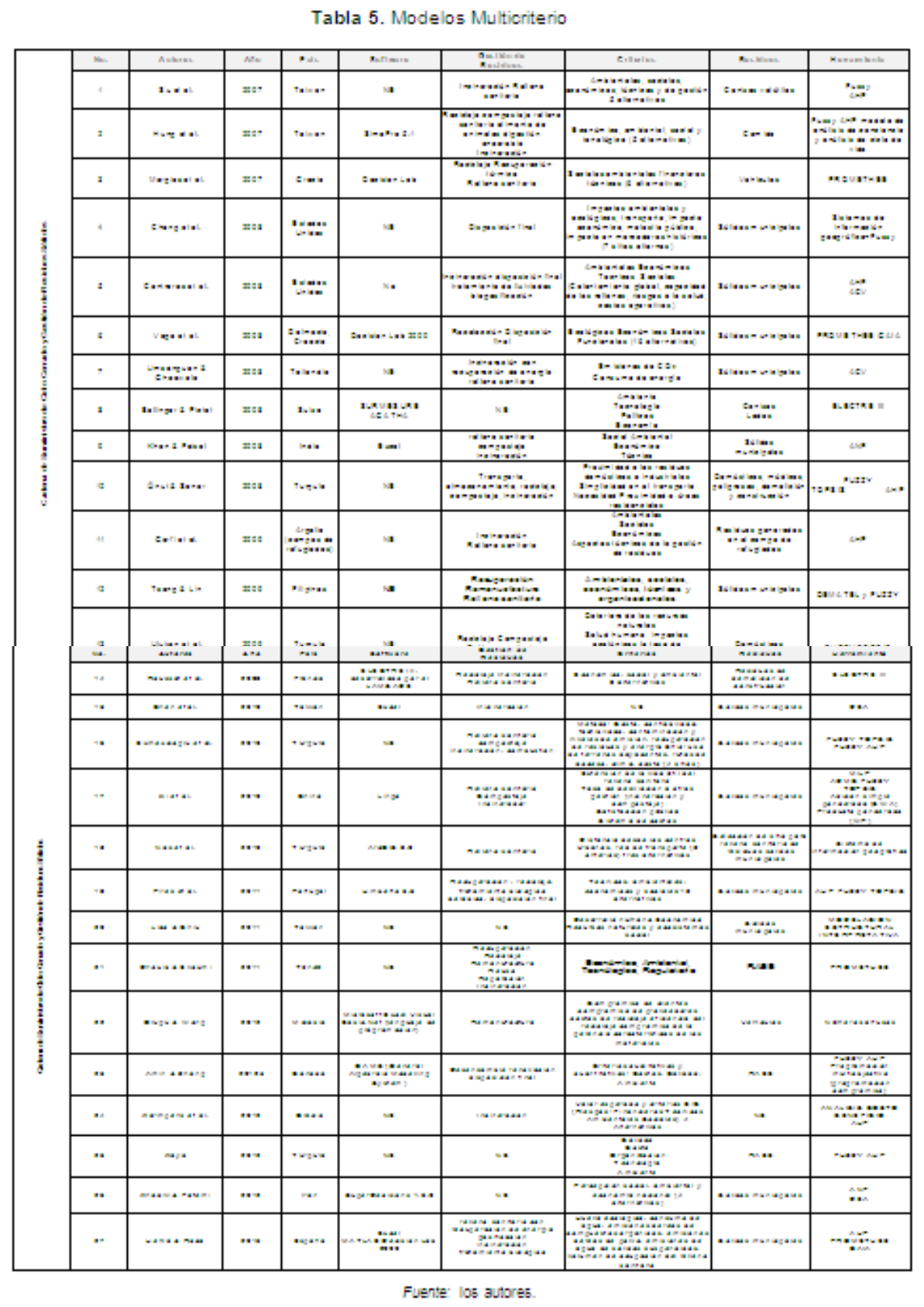

Hacer click en la imagen para ampliarla 
Tabla 6. Convenciones usadas en la técnica multicriterio

\begin{tabular}{ll}
\hline ACRONIMO & SIGNIFICADO \\
\hline ACV & Análisis del Ciclo de Vida \\
AHP & Proceso Analítico Jerárquico \\
ANP & Proceso Analítico de Redes \\
DEA & Análisis Envolvente de Datos \\
DEMATEL & Decision Making Trial and Evaluation Laboratory \\
ELECTREE & Limination Et Choix Traduisant la Realité \\
FUZZY & Difuso \\
GAIA & Geometrical Analysis for Interactive Aid \\
NE & No especifica \\
PROMETHEE & Preference Ranking Organisation METHod for Enrichment Evaluations \\
QFD & Quality Function Deployment \\
RAEE & Residuos de aparatos eléctricos y electrónicos \\
TOPSIS & Tecnnique for Order Performance by Similarity to Ideal Soluction \\
\hline
\end{tabular}

Tabla 7. Frecuencia de artículos por año de publicación

\begin{tabular}{lccc}
\hline \multicolumn{1}{c}{ Años } & Optimización & Simulación & Multicriterio \\
\hline 2003 & & 2 & \\
2007 & & 1 & 3 \\
2008 & & 1 & 7 \\
2009 & 1 & & 4 \\
2010 & 3 & 2 & 4 \\
2011 & 3 & 1 & 3 \\
2012 & 6 & & 5 \\
2013 & & & 1 \\
\hline
\end{tabular}

Fuente: Ios autores. 
Tabla 8. Frecuencia de artículos por tipo de residuo

\begin{tabular}{lccc}
\hline \multicolumn{1}{c}{ Residuos } & Optimización & Simulación & Multicriterio \\
\hline NE & 5 & 3 & 1 \\
Vidrio & 1 & & \\
Baterias & 1 & & \\
varios & 1 & & 1 \\
RAEE & 5 & 3 & 3 \\
Zinc & & 1 & 2 \\
Vehiculos & & & 2 \\
Demolición & & & 2 \\
Cenizas & & & 1 \\
Comida & & & 15 \\
Solidos municipales & & & \\
\hline & & & \\
RAEE: Residuos de & aparatos eléctricos y
\end{tabular}

electrónicos. NE: No especifica.

Fuente: Ios autores.

Tabla 9. Frecuencia de artículos por tipo de herramienta usada

\begin{tabular}{lccc} 
Herramientas & Optimización & Simulación & Multicriterio \\
\hline MILP & 4 & & \\
HIBRIDO MILP & 5 & & \\
HIBRIDO MOB & 2 & & \\
MILNP & 1 & 5 & \\
DINAMICA SISTEMAS & & 1 & \\
OTRO & 1 & 1 & \\
EVENTOS DISCRETOS & & & 16 \\
HIBRIDOS MULTICRITERIO & & & 2 \\
PROMETHEE & & & 1 \\
ACV & & & \\
ELECTRE & & & 1 \\
AHP & & & 1 \\
ANP & & & 1 \\
MEI & & & 1 \\
SIG & & \\
DEA & & \\
FUZZY & &
\end{tabular}

Fuente: Ios autores. 
Tabla 10. Frecuencia de artículos por tipo de software usado

\begin{tabular}{|c|c|c|c|}
\hline Software & Optimización & Simulación & Multicriterio \\
\hline LINGO & 1 & & \\
\hline LINDO & 2 & & \\
\hline GAMS & 5 & & \\
\hline CPLEX & 4 & & \\
\hline SLAM & 1 & & \\
\hline VENSIM & & 1 & \\
\hline POWERSIM & & 3 & \\
\hline QUEST & & 1 & \\
\hline ASPEN PLUS & & 1 & \\
\hline NE & & 1 & 13 \\
\hline DECISION LAB & & & 3 \\
\hline SUPERDECISION & & & 1 \\
\hline EXCEL & & & 2 \\
\hline SIMAPRO & & & 2 \\
\hline UMBERTO & & & 1 \\
\hline OTROS & & & 5 \\
\hline
\end{tabular}

NE: No especifica.

Fuente: Ios autores.

Tabla 11. Frecuencia de artículos por país de procedencia

\begin{tabular}{lccc}
\hline País & Optimización & Simulación & Multicriterio \\
\hline Canadá & 4 & & 1 \\
Turquia & 3 & & 5 \\
Portugal & 2 & & 1 \\
Irán & 2 & & 1 \\
India & 2 & 3 & 1 \\
Grecia & & 2 & 2 \\
Alemania & & 2 & \\
Japón & & \\
Taiwan & & 4 \\
Estados Unidos & & 2 \\
España & & 1 \\
Francia & & 1 \\
Suiza & & 1 \\
Croacia & & 1 \\
Argelia & & 1 \\
Tailandia & & 1 \\
Filipinas & & 1 \\
Malasia & & 1 \\
Túnez & & 1 \\
China & & \\
\hline
\end{tabular}

Fuente: los autores.

Por otra parte, se destaca la orientación hacia el estudio de los RAEE como fotocopiadoras y computadores, porque en regiones como la Unión Europea existen regulaciones específicas para estos materiales las cuales obligan a los productores a tomar responsabilidad por sus productos al final de la vida útil. Además, tienen impactos ambientales por componentes como el arsénico 
y plomo en los circuitos electrónicos (Talbot et al., 2007) y por el incremento exponencial en la generación de residuos electrónicos (Souza, 2013).

\section{Coocurrencia de palabras}

La aplicación del software TLAB, permitió identificar 165 palabras diferentes de los 47 artículos seleccionados y 28 de ellas se denominaron palabras clave por tener frecuencia mayor a cuatro. Este software permite conocer la frecuencia y la coocurrencia de las palabras, especifíca las relaciones anteriores y posteriores de cada palabra y las intersecciones entre frases y palabras. Así por ejemplo, la palabra con mayor frecuencia fue waste con 22 apariciones seguido de supply, chain, management, closed loop y solid.

Al identificar las relaciones entre palabras con la ayuda de la Figura 2A, se observó que las coocurrencias más comunes con la palabra supply eran chain, reverse, logistic, uncertainty, closed-loop, network y optimization y las más alejadas eran waste, dinamics, solid y multicriteria. Esta tendencia confirma que los asuntos que más interesan en la decisiones en las CSCC son aquellos relacionados con el diseño de la red, la consideración de dimensiones como la LR y de la incertidumbre relacionada con la frecuencia, cantidad y calidad de los materiales retornados, la cual se aborda desde modelos de optimización.

En la Figura 2B se muestra que la palabra compuesta closedloop presentó el mayor número de coocurrencias con la palabra chain, supply, uncertainty, optimization, reverse y logistic y guardaba poca relación con palabras como waste, solid, municipal, management y multicriteria. En este sentido, la teoría en torno a las decisiones en las CSCC se orienta hacia minimización de costos o maximización de utilidad y la mayoría presenta objetivos estratégicos como el diseño de la CSCC bajo modelos de optimización. Además, las investigaciones se han concentrado en la gestión de retornos como en el caso de productos defectuosos.

La palabra waste en la Figura 2C, presentó coocurrencia con municipal, life-cycle, sustainable, fuzzy, multicrieria, decision making y solid; sin embargo, la coocurrencia fue baja con palabras como closed-loop, supply, chain, dinamics y uncertainty y no hubo relación alguna con la palabra optimization. Se identifica la necesidad de desarrollar más investigaciones basadas en la experiencia y respaldada con modelos de optimización (Souza, 2013) y se confirma la baja relación de los temas de gestión de residuos con las decisiones en la CSCC; como lo afirman De Brito y van der Laan (2010), las decisiones en las cadenas de suministro están muy vinculadas con aspectos operativos y no con ambientales o sociales. 


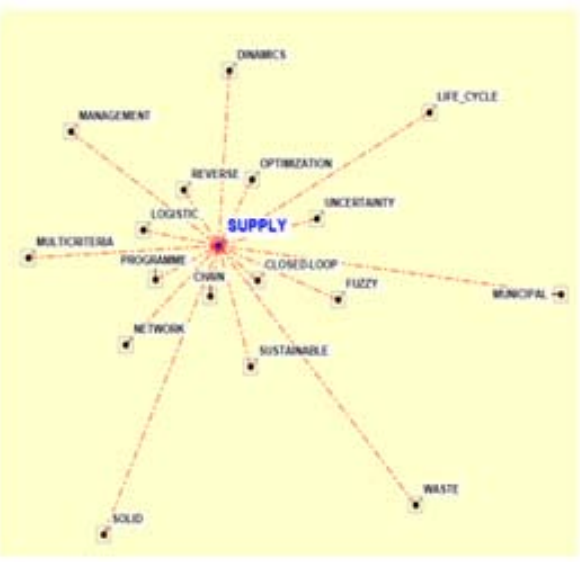

A. Supply

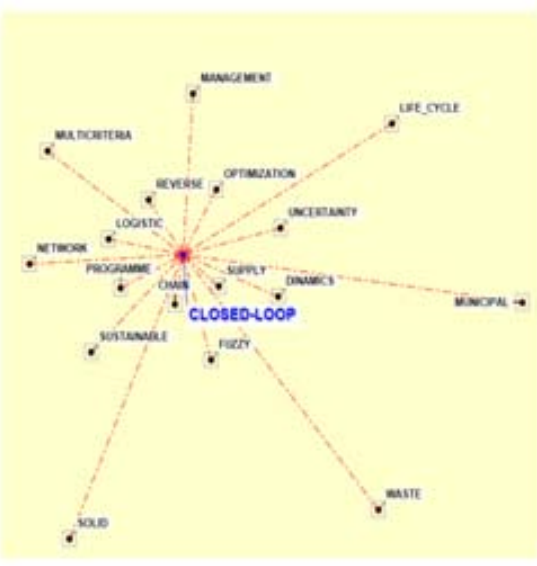

B. Closed-loop

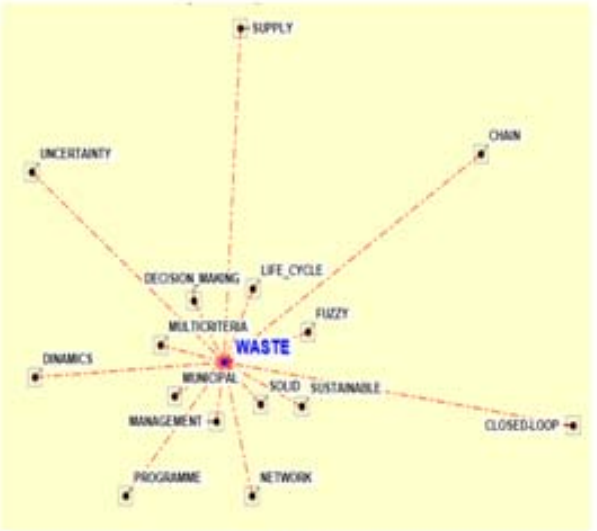

\section{Waste}

Fuente: los autores.

Figura 2. Coocurrencias de palabras.

Hacer click en la imagen para ampliarla

Además, debido a la complejidad del flujo de residuos y el aumento en las producción de ellos, el proceso de toma de decisiones para la efectiva GIRS requiere de la consideración de criterios en ocasiones conflictivos, lo cual ha sido abordado principlamente desde las diferentes herramientas de la técnica de análisis multicriterio (Achillas et al., 2013); así mismo, se observa que el flujo de residuos más investigado es de los residuos sólidos municipales. En general se presenta poca relación entre los asuntos de gestión de residuos sólidos y las cadenas de suministro, sean estas directas, reversas o de ciclo cerrado.

Con base en el análisis realizado y en la búsqueda rigurosa de documentos que relacionaran experiencias colombianas en los temas del estudio, se encontró que algunos estudios se refieren a las dimensiones de la CSCC (LV y LR); sin embargo ninguno se relacionaba con CSCC y GIRS en el contexto local. Como lo señalaron Peña et al. (2013), es necesario fortalecer la investigación que integre la gestión integral y sostenible de residuos sólidos en las cadenas de suministro, ya que las experiencias reportadas para la región se basan principalmente 
en prácticas de países desarrollados y se fundamentan en motivaciones económicas.

\section{Mapas tecnológicos}

El mapa tecnológico de interés para la investigación correpondió a la relación entre país, residuo y herramienta de modelación. En esta sección se agruparon los países por continentes y algunos residuos, como por ejemplo, fotocopiadoras y computadores se clasificaron en la categoría de RAEE. En el cuadrante inferior izquierdo de la Figura $3 \mathrm{~A}$ se puede observar que los países de América (Canadá y Estados Unidos) investigan en RAEE o en ocasiones no especifican un residuo en la investigación y emplean principalmente la herramienta de optimización.

De igual forma, en la parte derecha de la Figura 3A se observa que la técnica multicriterio se usa principalmente para investigar en residuos sólidos municipales, cenizas, demolición de construcciones y vehículos; los países asiáticos se relacionaron con esta tendencia.

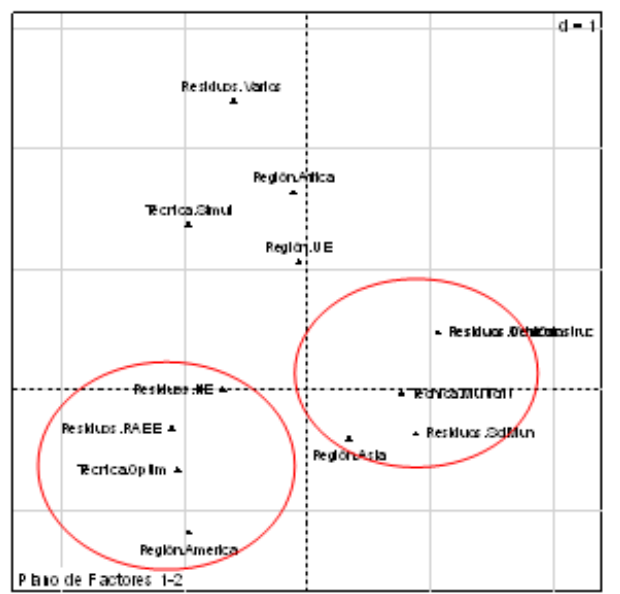

A. Plano de factores 1-2

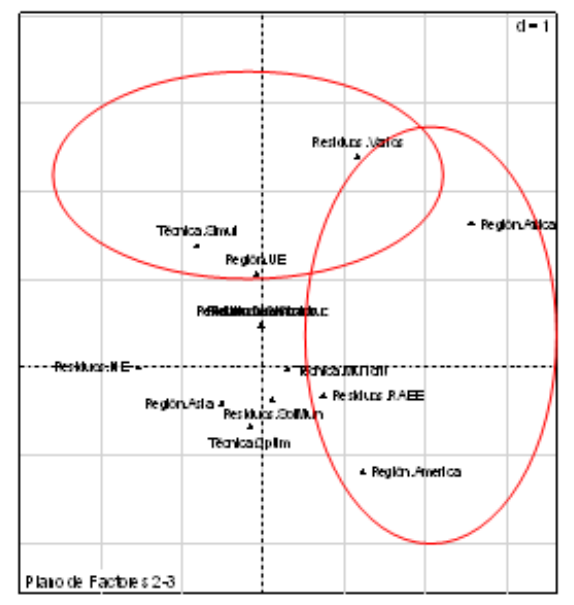

B. Plano de factores 2-3

Fuente: Ios autores.

Figura 3. Mapa tecnológico país, residuos, herramienta.

Hacer click en la imagen para ampliarla

Otro plano del análisis se aprecia en la Figura 3B parte superior, en la cual se muestra que los países de la Unión Europea investigan en residuos varios (como peligrosos y hospitalarios) y usan principalmente la herramienta de simulación. En la parte superior derecha de la Figura 3B, se observa que los países africanos presentan relación con los países americanos en cuanto la investigación en RAEE; sin embargo, se da esta relación por el bajo número de artículos provenientes de países africanos, mientras que los americanos tienen más alta participación en número y estudios en RAEE. 
Cabe destacar que la información presentada en los mapas tecnológicos tiende a estar dispersa debido al número reducido de artículos y la variedad de temas que se consideran en ellos. Los mapas tecnológicos brindan información más precisa a mayor cantidad y frecuencia de la información; no obstante, este análisis constituye un panorama del desarrollo de los temas centrales de esta investigación y las oportunidades para futuras investigaciones.

\section{CONCLUSIONES}

El análisis realizado muestra que todas las técnicas son aplicables para la la GIRS en CSCC; sin embargo, la de mayor aplicación es la multicriterio. Las técnicas multicriterio abordan situaciones complejas al involucrar aspectos tanto cualitativos como cuantitativos de la GRS; en este sentido consideran gran variedad de criterios como los económicos, ambientales, sociales, técnicos y culturales con el objeto de incluir la mayoría de aspectos que se presentan en la GRS. Los criterios más comunes suelen ser los económicos y los ambientales.

Particularmente, el uso de técnicas de análisis multicriterio se ha explorado poco en las cadenas de suministro y su aporte se refleja significativamente para la gestión de residuos sólidos municipales. Esta técnica contribuiría a la definición de aquellos criterios que enfrentan las CSCC en países en desarrollo, a través de la integración del juicio de expertos académicos y empresariales que contribuyan a explorar estas iniciativas.

Se identifica también el potencial de aplicación de técnicas de optimización y de simulación que existen en en el contexto colombiano para la GIRS en las CSCC. La exploración de estas técnicas en estos sistemas constituye una oportunidad muy importante de investigación dadas la regulaciones ambientales que se están implementando y la responsabilidad en la toma de decisiones respecto a un objeto de conocimiento que se debate entre las demandas del consumo y la calidad ambiental relacionada con el descarte de residuos sólidos.

\section{AGRADECIMIENTOS}

Los autores agradecen a la Universidad del Valle por la financiación del proyecto denominado "Modelo soporte a la toma de decisiones para la gestión integral y sostenible de residuos sólidos en cadenas de suministro de ciclo cerrado del Valle del Cauca" Agosto 2012-Agosto 2014. 


\section{REFERENCIAS}

- Achillas, C., Vlachokostas, C., Aidonis, D., Moussiopoulos, lakovou, E. y Banias, G. (2010). Optimising reverse logistics network to support policy-making in the case of Electrical and Electronic Equipment. Waste Management, 30(12), 2592-2600.

- Achillas, C., Moussiopoulos, N., Karagiannidis, A., Banias, G. y Perkoulidis, G. (2013). The use of multi-criteria decision analysis to tackle waste management problems: a literature review. Waste Management \& Research, 31(2), 115-129.

- Amin, S.H. y Zhang, G. (2012a). An integrated model for closedloop supply chain configuration and supplier selection: Multiobjective approach. Expert Systems with Applications, 39(8), 6782-6791.

- . (2012b). A proposed mathematical model for closedloop network configuration based on product life cycle, The International Journal of Advanced Manufacturing Technology, 58, 791-801.

- Amin, S.H. y Zhang, G. (2013). A multi-objective facility location model for closed-loop supply chain network under uncertain demand and return. Applied Mathematical Modelling, 37(6), 41654176.

- Aragonés-Beltrán, P., Pastor-Ferrando, J. P., García-García, F. y Pascual-Agulló, A. (2010). An analytic network process approach for siting a municipal solid waste plant in the metropolitan area of Valencia (Spain). Journal of Environmental Management, 91(5), 1071-1086.

- Banar, M., Özkan, A. y Kulaç, A. (2010). Choosing a recycling system using ANP and ELECTRE III techniques. Turkish J Eng Env Sci, 34, 145-154.

- Barker, T.J. y Zabinsky, Z.B. (2011). A multicriteria decision making model for reverse logistics using analytical hierarchy process. Omega, 39(5), 558-573.

- Besiou, M., Georgiadis, P. y Van Wassenhove, L. N. (2012). Official recycling and scavengers: Symbiotic or conflicting? European Journal of Operational Research, 218(2), 563-576.

- Biehl, M., Prater, E. y Realff, M.J. (2007). Assessing performance and uncertainty in developing carpet reverse logistics systems. Computers \& Operations Research, 34(2), 443-463.

- Bollinger, D. y Pictet, J. (2008). Multiple criteria decision analysis of treatment and land- filling technologies for waste incineration residues. Omega, 36(3), 418-428.

- Casas, J.J. y Cerón, K. (2013). Método cuantitativo para la configuración de la cadena de logística inversa de un tipo de residuo de aparato eléctrico o electrónico. Trabajo de grado, Ingeniería Industrial, Universidad del Valle. Cali.

- Chang, N.B., Parvathinathan, G. y Breeden, J.B. (2008). Combining GIS with fuzzy multicriteria decision-making for landfill siting in a fast-growing urban region. Journal of environmental management, 87(1), 139-153

- Chen, C.T., Lin, C.T. y Huang, S.F. (2006). A fuzzy approach for supplier evaluation and selection in supply chain management. International journal of production economics, 102(2), 289-301.

- Contreras, F., Hanaki, K., Aramaki, T. y Connors, S. (2008). Application of analytical hierarchy process to analyze stakeholders 
preferences for municipal solid waste management plans, Boston, USA. Resources, Conservation and Recycling, 52(7), 979-991.

- Dale, R., Melamed, B. y Lembke, R. (2012). Modeling and Analysis of Reverse Logistics. Journal of Business Logistics, 33(2), 107-117.

- De Brito, M.P. y van der Laan, E. A. (2010). Supply Chain Management and Sustainability: Procrastinating Integration in Mainstream Research. Sustainability, 2(4), 859-870.

- Demirel, N.Ö. y Gökçen, H. (2008). A mixed integer programming model for remanufacturing in reverse logistics environment. The International Journal of Advanced Manufacturing Technology, 39(11-12), 1197-1206.

- Dhouib, D. y Elloumi, S. (2011). A new multi-criteria approach dealing with dependent and heterogeneous criteria for end-of-life product strategy. Applied Mathematics and Computation, 218(5), 1668-1681.

- Ekmekçioglu, M., Kaya, T. y Kahraman, C. (2010). Fuzzy multicriteria disposal method and site selection for municipal solid waste. Waste Management, 30(8), 1729 -1736.

- Escorsa, P. y Maspons, R. (2001). De la vigilancia tecnológica a las inteligencia competitiva. Madrid: Ed. Financial Times - Prentice Hall (Grupo Pearson).

- Ferguson, M.E. y Souza, G.C. (2010). Closed-loop supply chains: new developments to improve the sustainability of business practices. CRC Press.

- Figueira, J., Greco, S. y Ehrgott, M. (2005). Multiple criteria decision analysis: state of the art surveys. Vol. 78. Springer.

- Fleischmann, M., Krikke, H.R., Dekker, R. y Flapper, S.D.P. (2000). A characterisation of logistics networks for product recovery. Omega, 28(6), 653-666.

- Fröhling, M., Schwaderer, F., Bartusch, H. y Rentz, O. Integrated planning of transportation and recycling for multiple plants based on process simulation. European Journal of Operational Research, 207, 958-970.

- Garfi, M., Tondelli, S. y Bonoli, A. (2009). Multi-criteria decision analysis for waste management in Saharawi refugee camps. Waste management, 29(10), 2729-2739.

- Georgiadis, P. y Besiou, M. (2008). Sustainability in electrical and electronic equipment closed-loop supply chains: A System Dynamics approach, Journal of Cleaner Production, 16, 16651678.

- Gomes-Salema, M.I., Barbosa-Povoa, A.P. y Novais, A.Q. (2007). An optimization model for the design of a capacitated multiproduct reverse logistics network with uncertainty. European Journal of Operational Research, 179(3), 1063-1077.

- . (2010). Simultaneous design and planning of supply chains with reverse flows: A generic modelling framework. European journal of operational research, 203(2), 336-349.

- Granada-Aguirre, L.F. (2009). Gestión ambiental empresarial: Pasado, presente y futuro de las normas e instituciones ambientales en Colombia. Libre Empresa, 6(1), 63-79.

- Herva, M., y Roca, E. (2013). Ranking municipal solid waste treatment alternatives based on ecological footprint and multicriteria analysis. Ecological Indicators, 25, 77-84. 
- Hung, M.L., Ma, H. y Yang, W.F. (2007). A novel sustainable decision making model for municipal solid waste management. Waste Management, 27(2), 209-219.

- Jianghong, M. (2010). An Integrated Supply Chain Modeling and Simulation Based on System Dynamics. Paper presented at the Management and Service Science (MASS).

- Kannan, G., Sasikumar, P. y Devika, K. (2010). A genetic algorithm approach for solving a closed loop supply chain model: A case of battery recycling. Applied Mathematical Modelling, 34(3), 655-670.

- Karmperis, A.C., Sotirchos, A., Aravossis, K. y Tatsiopoulos, I.P. (2012). Waste management project's alternatives: A risk-based multi-criteria assessment (RBMCA) approach. Waste management, 32(1), 194-212.

- Kaya, I. (2012). Evaluation of outsourcing alternatives under fuzzy environment for waste management. Resources, Conservation and Recycling, 60, 107-118.

- Khadivi, M.R. y Fatemi Ghomi, S.M.T. (2012). Solid waste facilities location using of analytical network process and data envelopment analysis approaches. Waste management, 32(6), 1258-1265.

- Khan, S. y Faisal, M.N. (2008). An analytic network process model for municipal solid waste disposal options. Waste management, 28(9), 1500-1508.

- Lee, D.-H., Dong, M. y Bian, W. (2010). The design of sustainable logistics network under uncertainty. International Journal of Production Economics, 128(1), 159-166.

- Liamsanguan, C. y Gheewala, S.H. (2008). LCA: A decision support tool for environmental assessment of MSW management systems. Journal of Environmental Management, 87 (1), 132-138.

- Liao, C.H. y Chiu, A.S. (2011). Evaluate municipal solid waste management problems using hierarchical framework. ProcediaSocial and Behavioral Sciences, 25, 353-362.

- Lu, T. y Zhao, X. (2010). Reverse Logistics Network Design with Consideration of Forward and Reverse Facility Integration. Paper presented at the E-Business and E-Government (ICEE).

- Mergias, I., Moustakas, K., Papadopoulos, A. y Loizidou, M. (2007). Multi-criteria decision aid approach for the selection of the best compromise management scheme for ELVs: The case of Cyprus. Journal of Hazardous Materials, 147(3), 706-717.

- Ministerio de Vivienda, Ciudad y Territorio. (2013). Decreto 2981 "Por el cual se reglamenta la prestación del servicio de aseo". República de Colombia.

- Mitra, S. (2012). Inventory management in a two-echelon closedloop supply chain with correlated demands and returns. Computers \& Industrial Engineering, 62(4), 870-879.

- Nas, B., Cay, T., Iscan, F. y Berktay, A. (2010). Selection of MSW landfill site for Konya, Turkey using GIS and multi-criteria evaluation. Environmental monitoring and assessment, 160(1-4), 491-500.

- Olugu, E.U. y Wong, K.Y. (2012). An expert fuzzy rule-based system for closed-loop supply chain performance assessment in the automotive industry. Expert Systems with Applications, 39(1), 375-384. 
- Önüt, S. y Soner, S. (2008). Transshipment site selection using the AHP and TOPSIS approaches under fuzzy environment. Waste Management, 28(9), 1552-1559.

- Özkir, V. y Basligil, H. (2013). Multi-objective optimization of closed-loop supply chains in uncertain environment. Journal of Cleaner Production, 41, 114-125.

- Paksoy, T., Özceylan, E. y Weber, G. (2010). A multi objective model for optimization of a green supply chain network. AIP Conference Proceedings, 1239(1). p. 311.

- Paksoy, T. y Bektas, T. (2011). Operational and environmental performance measures in amulti-product closed-loop supply chain. Transportation Review. Part E: Logistics and Transportation Review, 47(4), 532-546.

- Paksoy, T., Bektas, T. y Özceylan, E. (2011). Operational and environmental performance measures in a multi-product closedloop supply chain. Transportation Research Part E: Logistics and Transportation Review, 47(4), 532-546.

- Peña Montoya, C.C., Torres Lozada, P., Vidal Holguín, C.J. y Marmolejo Rebellón, L.F. (2013). La logística de reversa y su relación con la gestión integral y sostenible de residuos sólidos en sectores productivos. Revista Entramado, 9(1), 226-238.

- Pires, A., Chang, N.-B. y Martinho, G. (2011). An AHP-based fuzzy interval TOPSIS assessment for sustainable expansion of the solid waste management system in Setúbal Peninsula, Portugal. Resources, Conservation and Recycling, 56(1), 7-21.

- Pishvaee, M.S., Jolai, F. y Razmi, J. (2009). A stochastic optimization model for integrated forward/reverse logistics network design. Journal of Manufacturing Systems, 28(4), 107-114.

- Pishvaee, M.S., Rabbani, M. y Torabi, S.A. (2011). A robust optimization approach to closed-loop supply chain network design under uncertainty. Applied Mathematical Modelling, 35(2), 637649.

- Queiruga, D., Walther, G., González-Benito, J. y Spengler, T. (2008). Evaluation of sites for the location of WEEE recycling plants in Spain. Waste Management, 28(1), 181-190.

- Ramezani, M., Bashiri, M. y Tavakkoli-Moghaddam, R. (2013). A new multi-objective stochastic model for a forward/reverse logistic network design with responsiveness and quality level. Applied Mathematical Modelling, 37(1), 328-344.

- Rogers, D.S. y Tibben-Lembke, R. S. (1998). Reverse Logistics and the Environment. En RLEC (Ed.), Going Backwards:Reverse Logistics Trends and Practices (pp. 101-136).

- Rousis, K., Moustakas, K., Malamis, S., Papadopoulos, A. y Loizidou, M. (2008). Multi-criteria analysis for the determination of the best WEEE management scenario in Cyprus. Waste Management, 28(10), 1941-1954.

- Roussat, N., Dujet, C. y Mehu, J. (2009). Choosing a sustainable demolition waste management strategy using multicriteria decision analysis. Waste Management, 29(1), 12-20.

- Senthil, S., Srirangacharyulu, B. y Ramesh, A. (2012). A decision making methodology for the selection of reverse logistics operating channels. Procedia Engineering, 38, 418-428.

- Shapiro, J.F. (2007). Modeling the supply chain. Vol. 2. Cengage Learning.

- Shi, Y., Wang, J. y Xiao, S. (2010). Research on reverse logistics network design for television remanufacturing based on operation 
research method and simulation. Paper presented at the 2010 IEEE 17th International Conference on Industrial Engineering and Engineering Management.

- Soma, M., Kondoh, S. y Umeda, Y (2003). Simulation of closedloop manufacturing systems focused on material balances in Environmentally Conscious Design and Inverse Manufacturing. EcoDesign '03. pp. 95-101.

- Souza, G.C. (2013). Closed Loop Supply Chains: A Critical Review, and Future Research. Decision Sciences, 44(1), 7-38.

- Spengler, T. y Schröter, M. (2003). Special Section: Closed-Loop Supply Chains: Practice and Potential: Strategic Management of Spare Parts in Closed-Loop Supply Chains - A System Dynamics Approach. Interfaces, 33, 7-17.

- Stindt, D. y Sahamie, R. (2014). Review of research on closed loop supply chain management in the process industry. Flexible Services and Manufacturing Journal, 26(1-2), 268-293.

- Su, J.P., Chiueh, P.T., Hung, M.L. y Ma, H.W. (2007). Analyzing policy impact potential for municipal solid waste management decision-making: A case study of Taiwan. Resources, Conservation and Recycling, 51(2), 418-434.

- Tako, A. y Robinson, S. (2012). The application of discrete event simulation and system dynamics in the logistics and supply chain context. Decision Support Systems, 52(4), 802-815.

- Talbot, S., Lefebvre, É. y Lefebvre, L. (2007). Closed-loop supply chain activities and derived benefits in manufacturing SMEs. Journal of Manufacturing Technology Management, 18(6), 627658.

- Thiell, M., Soto, J.P., Madiedo, J.P. y van Hoof, B. (2011). Green Logistics: Global Practices and their Implementation in Emerging Markets. Green Finance and Sustainability. Business Science Reference, 334-357.

- Ulukan, H.Z. y Kop, Y. (2009). Multi-criteria decision making (MCDM) of solid waste collection methods using life cycle assessment (LCA) outputs. In Computers \& Industrial Engineering, 2009. CIE 2009. pp. 584-589.

- Vego, G., Kucar-Dragicevic, S. y Koprivanac, N. (2008). Application of multi-criteria decision-making on strategic municipal solid waste management in Dalmatia, Croatia. Waste Management, 28(11), 2192-2201

- Vlachos, D., Georgiadis, P. y lakovou, E. (2007). A system dynamics model for dynamic capacity planning of remanufacturing in closed-loop supply chains. Computers \& Operations Research, 34, 367-394.

- Wang, F., Lai, X. y Shi, N. (2011). A multi-objective optimization for green supply chain network design. Decision Support Systems, 51(2), 262-269.

- Wang, L. y Murata T. (2011). Study of Optimal Capacity Planning for Remanufacturing Activities in Closed-Loop Supply Chain using System Dynamics Modeling Proceeding of the IEEE International Conference on Automation and Logistics Chongqing, China.

- Xi, B.D., Su, J., Huang, G.H., Qin, X.S., Jiang, Y.H., Huo, S.L. y Yao, B. (2010). An integrated optimization approach and multicriteria decision analysis for supporting the waste-management system of the City of Beijing, China. Engineering Applications of Artificial Intelligence, 23(4), 620-631. 
- Xianfeng, L., Jianwei, Q. y Meilian, L. (2010). Design and simulation WEEE reverse logistics network in Guangxi. Paper presented at the Optoelectronics and Image Processing (ICOIP).

- Yeomans, J.S. (2007). Solid waste planning under uncertainty using evolutionary simulation-optimization. Socio-Economic Planning Sciences, 41(1), 38-60.

- Zeballos, L.J., Gomes, M.I., Barbosa-Povoa, A.P. y Novais, A.Q. (2012). Addressing the uncertain quality and quantity of returns in closed-loop supply chains. Computers \& Chemical Engineering, 47, 237-247.

- Zhang, G. y Amin, S.H. (2011). Network design of a closed-loop supply chain with uncertain demand and return. In Service Operations, Logistics, and Informatics (SOLI), 376-379.

- Zhou, Y. y Wang, S. (2008). Generic model of reverse logistics network design. Journal of Transportation Systems Engineering and Information Technology, 8(3), 71-78.

1. Ingeniera Industrial. MSc. in Management. Candidata Doctorado en Ingeniería, énfasis Ingeniería Sanitaria y Ambiental, Universidad del Valle. claudia.pena@correounivalle.edu.co

2. Ingeniero Industrial. Magíster en Ingeniería Industrial. Profesor Titular Escuela de Ingeniería Industrial, Universidad del Valle. juan.osorio@correounivalle.edu.co

3. Ingeniero Mecánico. Ph.D. y MSc. en Ingeniería Industrial. Profesor Titular Escuela de Ingeniería Industrial, Universidad del Valle.carlos.vidal@correounivalle.edu.co

4. Ingeniera Sanitaria. Ph.D. y MSc. en Ingeniería Civil, énfasis Hidráulica y Saneamiento. Profesora Titular Escuela EIDENAR, Universidad del Valle. patricia.torres@correounivalle.edu.co

5. Ingeniero Sanitario. Doctor en Ingeniería, énfasis Ingeniería Sanitaria y Ambiental, Universidad del Valle. Profesor Asistente Escuela EIDENAR. Universidad del Valle. luis.marmolejo@correounivalle.edu.co

Para citar este artículo: Peña-Montoya, C.C., Osorio-Gómez, J.C., Vidal-Holguín, C.J. Torres-Lozada, P. y MarmolejoRebellón, L.F. (2014). Gestión de residuos sólidos en cadenas de suministro de ciclo cerrado desde la perspectiva de la investigación de operaciones. Revista Luna Azul, 41, 05-28. Recuperado de http://lunazul.ucaldas.edu.co/index.php?option=content\&tas k=view\&id=1053 